Journal of Engineering and Technology Research Vol. 5(2), pp. 33-41, February 2013

Available online at http:// www.academicjournals.org/JETR

DOI: 10.5897/JETR2012.0194

ISSN 2006-9790 @2013 Academic Journals

Full Length Research Paper

\title{
Design and simulation study of a simple ion injector
}

\author{
M. M. Abdelrahman*, N.I. Basal and S.G. Zakhary \\ Accelerators and Ion Sources Department, Nuclear Research Center, Cairo Egypt.
}

Accepted 23 January, 2012

\begin{abstract}
The stated purpose of this manuscript is to study the parameters of a single-gap accelerator system with a view to optimizing the transport of a beam of argon ions. A study is made for the main factors that must be considered on the design of an ion injector taking into account the appropriate design of the single gap accelerator column by doing such study both analytically and with ion beam simulation. These study shows the dependence of the optimal voltage ratio which is related to the minimum beam emittance depends on the column dimension. Study of analytical calculations was done without space charge while simulation processes with space charge effect. Study of optical ion beam properties for a single gap accelerating column was done by using a SIMION computer program. A series of simulation studies were made in order to determine optimum conditions for acceleration of space-chargedominated argon ion trajectories with incident energy of $50 \mathrm{keV}$ from a distance $150 \mathrm{~mm}$ of the ion source at constant gradient acceleration system.
\end{abstract}

Key words: SIMION computer program, single gap tube acceleration system, beam emittance, injector system.

\section{INTRODUCTION}

Along with the development of computer and calculation techniques, numerical simulation has been a main tool to assist design and research of ion beam system. With high accuracy and velocity, numerical computation can study the effects of ion extraction system on characteristics of ion beams such as ion beam emittance. Also, simulation of ion beam injection into the accelerating tube for appropriate design of tube radius which determines the accelerator acceptance to match the beam emittance of the beam that exits from the ion source. Also, the simulation of beam trajectory inside the accelerating column is necessary to avoid ion beam aberration such as crossover inside and outside of the accelerating tube. The applications of accelerators include various branches of science and technology, medical treatment, and industrial processing. Accelerator scientists proposed many advanced technologies to produce beams with qualities required for each application (Lee 2000; Humphires 2002; Prakash et al 2012). Computer modeling of charged particle beam is

*Corresponding author. E-mail: moustafa82003@yahoo-com important part in investigation of processes that take place in different electro-physical equipments and has been used for about 50 years (Litovko and Oks 2006). The aim of these simulations is always to investigate the beam quality, which is necessary to adjust the beam conditions for the required applications. In this work, beam simulation is carried out to reveal the influence of the space charge effect on both the beam emittance and beam diameter. Also, influence of the tube diameter on the ion beam quality (beam emittance and beam diameter) was studied. Indeed, the influence of both charge state of the argon and mass of different elements on beam emittance and beam diameter for the singly charged argon ion trajectories was investigated. Samples of ion beam simulation with potential distributions inside the accelerating column with different tube diameters of 15,20 , and $30 \mathrm{~mm}$ were performed.

\section{OPTIMIZATION OF THE ION BEAM CHARACTERISTICS (INTENSITY AND EMITTANCE) PRODUCED FROM THE ION SOURCE}

To keep aberration as low as possible, the aspect ratio $S$ 


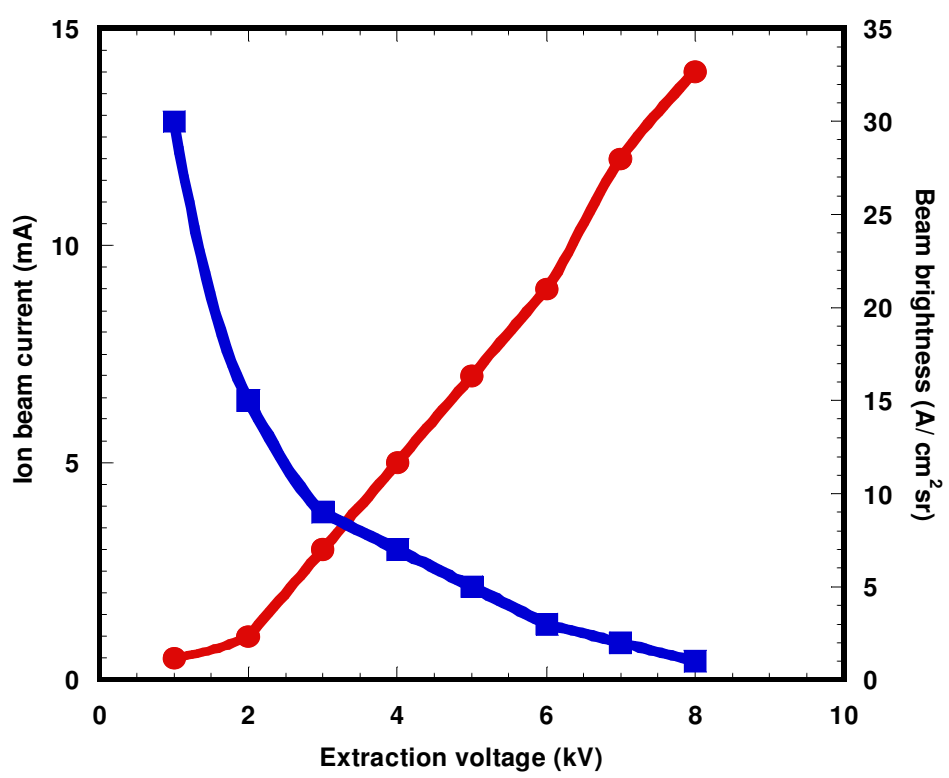

Figure 1. Influence of the extraction voltage on both the extracted ion current and beam brightness.

(the ratio of aperture radius to the electrode distance) should be of the order. $S=0.5$, with the breakdown limit in mind ( $d$ in $\mathrm{mm}, \mathrm{V}$ in $\mathrm{kV}$ ); $\mathrm{d} \geq 0.015 \mathrm{~V}^{1 / 2}$, the extracted current is given by (Spadtke 1992):

$$
I_{C h}=\frac{\pi}{9} \chi V^{3 / 2}
$$

Where

$$
\chi=2 \varepsilon_{0} \sqrt{\frac{2 q}{m}}
$$

On the other hand the emittance $\varepsilon$ can be estimated to first order by the aperture radius and a certain divergence angle of the beam if a waist is assumed at the exit of the extraction system:

$\varepsilon=\alpha r_{\circ}$

as mentioned before the aperture radius depends on the extraction voltage with the aspect ratio:

$\varepsilon=\alpha S d$, using the breakdown limit, the emittance can be estimated

$$
\varepsilon=2.37 \times 10^{-7} \alpha_{0} V^{3 / 2}
$$

The brightness is defined as, $\mathrm{B}=\mathrm{I} / \Omega \mathrm{A}$, where $\mathrm{I}$ is the current, $\mathrm{A}$ the area from which it is emitted, and $\Omega$ the solid angle into which the current is emitted.; we have (Spakudo and Hashi 1996):

$$
B=\frac{1.24 \times 10^{13} \varepsilon_{O} \sqrt{2 q / m}}{\alpha_{O}^{2} V^{3 / 2}}
$$

Figure 1 shows the influence of the extraction voltage on both the beam current and beam brightness extracted from hydrogen with assumed beam divergence $\alpha_{0}=20$ m.rad (Kunze et al 2003). It might be surprising that the current increases with the extraction voltage, whereas the brightness decreases with the extraction voltage. These dependences make clear why the optimization of extraction systems is necessary.

\section{ESTIMATION OF THE OPTIMAL VALUE OF THE ACCELERATING FIELD FOR BEST BEAM FOCUSING AND TRANSPORT WITHOUT ABERRATION}

Estimation of the optimal value of the accelerating field can be obtained from studying the motion of the ion beam through a single accelerating gap. From the study one can deduce the influence of the accelerating field on tracing the beam line within the gap and on the transverse beam emittance at the gap exit. The variation of the cross section of a beam propagating along the $Z$ axis of an accelerating column shown in Figure 2 can be determined using Equation 1 (Wilson and Brewer 1973, Abdelaziz et al 


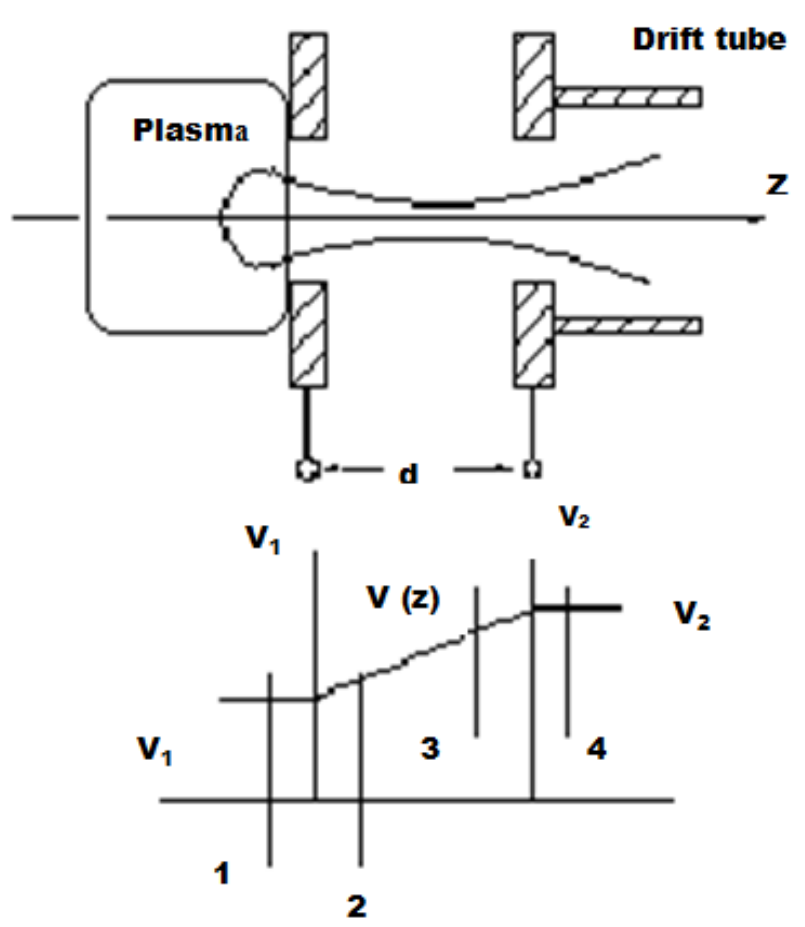

Figure 2. Schematic diagram of the single gap accelerating column with the variation of the potential distribution along the beam line.

1996, Abdelrahman and Zakhary 2011):

$\frac{\partial^{2} r}{\partial z^{2}}+\frac{V_{o}}{2 V_{o}} \frac{\partial r}{\partial z}+\frac{V_{o}}{4 V_{o}}=-Q \frac{r}{4 V_{o} \varepsilon_{o}}$

where $V_{0}$ is the potential on the $Z$ axis and the primes denotes the derivatives with respect to $Z$ axis, $Q$ represents the space charge density (which is assumed to be uniform

over the beam cross section ) and $\varepsilon_{0}$ is the dielectric permittivity of the free space. Neglecting the space charge effect, the right hand side of Equation 1 is set equal to zero. This equation was solved to determine the ray path between two planes representing the input and output of the accelerating column shown in Figure 2, located at a given distance apart (d) a long the $Z$ axis. Defining the input and output of an ion optical element $\left(r_{1}, r_{1}, V_{1}\right)$ refer to condition of beam radius, half angle of beam divergence, and the voltage at the input, $\left(r_{4}, r_{4}, V_{4}\right)$ are the conditions of the beam at the outlet. The solution is given by (Kirstein et al 1967, Reiser 1985):

Tracing the beam line within the gap (as shown in Figure 3) shows decrease of the beam outlet radius with the increase

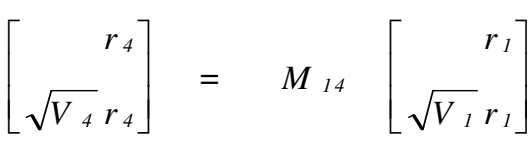

where

$$
M_{14}=\left[\begin{array}{cc}
\frac{3 \sqrt{V_{1}}-\sqrt{V_{2}}}{2 \sqrt{V_{1}}} & \frac{2 d}{\sqrt{V_{1}}+\sqrt{V_{2}}} \\
\frac{3\left(V_{2}-V_{1}\right)\left(\sqrt{V_{1}}-\sqrt{V_{2}}\right.}{8 d \sqrt{V_{1}} \sqrt{V_{2}}} & \frac{3 \sqrt{V_{1}}-\sqrt{V_{1}}}{2 \sqrt{V_{2}}}
\end{array}\right]
$$

of the accelerating ratio up to a certain optimal value. Higher than the optimal value the beam radius starts to increase and the aberration appears. Figure 3 shows the variation of the beam outlet radius with the accelerating ratio and Figure 3 also shows the variation of the transverse emittance of the beam outlet from a single gap with the voltage acceleration ratio applied to the gap. The optimal value of the accelerating ratio is found at the minimum beam emittance and with the beam transport without aberration (cross over).

\section{ION BEAM SIMULATION FOR SINGLE GAP ACCELERATION COLUMN}

A series of simulation studies were made in order to determine optimum conditions for acceleration of spacecharge-dominated argon gas with different incident energies at constant gradient acceleration system. The curvature of the plasma-emission surface tends to converge/diverge extracted ion beams. The optimum radius of curvature can be achieved by carefully balancing the plasma density in relation to the strength of the electric field. Under this condition, the angular divergence is minimum. As clearly illustrated, the curvature of the ion-emission boundary is critically dependent on the position of the extraction electrode relative to the emission aperture for fixed plasma density, charge-state distribution, and extraction-potential difference. The space charge force acts as a diverging force because particles of the same charge repel each other. The influence of space charge effects for both the quality and intensity of the extracted ion beams.

SIMION 3 D Version 7.0 (Dahl 2000, Dahl et al 1990) is a software package primarily used to calculate electric fields and the trajectories of charged particles in those fields when given a configuration of electrodes with voltages and particle initial conditions, including optional RF (quasistatic), magnetic field, and collisional effects. This program provides extensive supporting functionality 

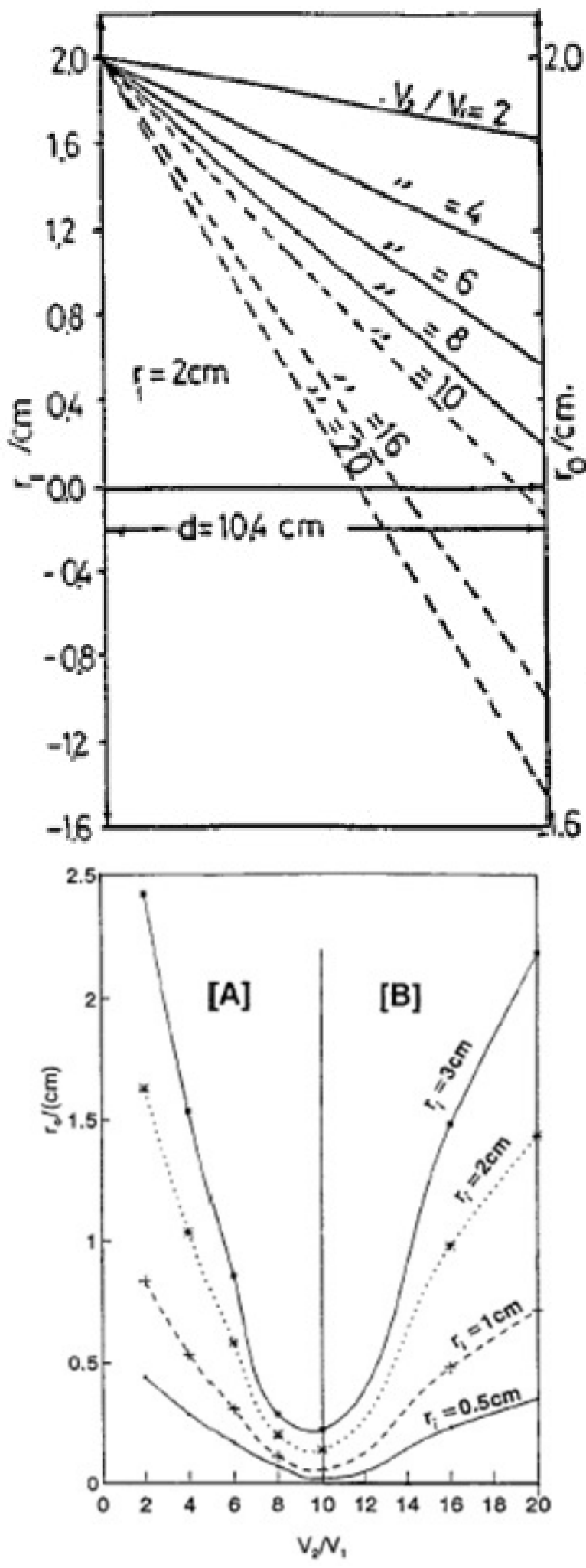

Figure 3. Schematic diagram of the single gap accelerating column with the variation of the potential distribution along the beam line 
Table 1. Relation between voltage ratio and beam emittance and beam radius for a single gap accelerating column.

\begin{tabular}{ccc}
\hline Voltage ratio $\left(\mathbf{V}_{\mathbf{2}} / \mathbf{V}_{\mathbf{1}}\right)$ & Beam emittance/ $\mathbf{1 0}(\mathbf{c m} \mathbf{~ m r a d})$ & Beam radius $(\mathbf{m m})$ \\
\hline 2 & 0.19 & 7.39 \\
4 & 0.04 & 6.12 \\
6 & 0.15 & 3.65 \\
8 & 0.38 & 0.68 \\
10 & 2.17 & 8.64 \\
12 & 7.30 & 20.95 \\
14 & 26.91 & 41.75 \\
16 & 87.36 & 83 \\
\hline
\end{tabular}

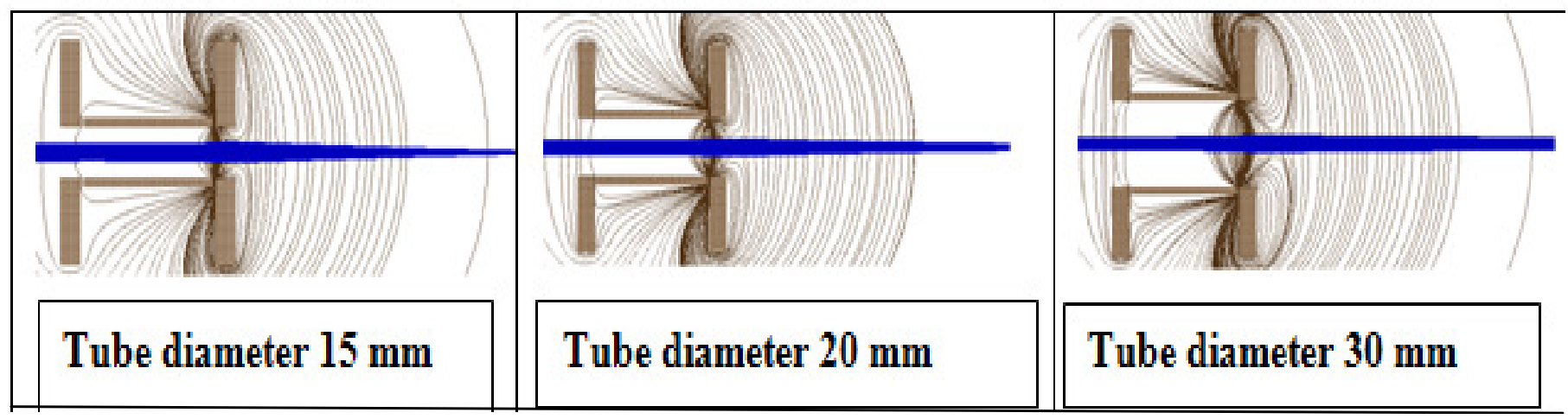

Figure 4. Single gap accelerating column as simulated by SIMION computer program for different tube diameters.

in geometry definition, user programming, data recording, and visualization. Simulation of the singly charged argon ion trajectories for flat plasma was studied with and without space charge effect using a model of constant gradient acceleration system with the aid of SIMION computer program. The simulation process was carried out at an assumption of a constant plasma density. In this work, beam simulation is carried out to reveal the influence of the space charge effect on the beam emittance and beam radius. Also, influence of the incident energy on the ion beam quality (beam emittance and beam radius) was studied. Indeed, the influence of the tube diameter on beam emittance for the singly charged argon ion trajectories was investigated.

Table 1 shows the relation between the voltage ratio applied to the single gap accelerating column system and both beam emittance and beam radius for a single charged argon ions with incident energy of $50 \mathrm{keV}$ from a distance $150 \mathrm{~mm}$ of the ion source. The first one of the single gap accelerating column was applied on a fixed voltage of $5 \mathrm{kV}$; where the second one was changed according to obtain several of voltage ratios of $2,4,6$, $8,10,12,14$ and 16 . It is clear from this table, minimum beam emittance was found at voltage ratio of 4 (output voltage applied is $20 \mathrm{kV}$ ) whereas maximum one at voltage ratio of 16 (output voltage applied is $80 \mathrm{kV}$ ). Also, minimum beam radius was found at voltage ratio of 8 (output voltage applied $40 \mathrm{kV}$ ), whereas maximum one at 16 (output voltage applied is $80 \mathrm{kV}$ ).

Sample of ion beam simulation inside the accelerating column with different input diameters of 15,20 , and $30 \mathrm{~mm}$ (Figure $4 \mathrm{a}, \mathrm{b}, \mathrm{c}$ ) were performed. This figure shows that the influence of variation of input tube diameter on both the beam emittance and beam radius for the argon ion trajectories passed through the single gap accelerating column system. It was seen that from this figure, minimum of beam emittance and beam radius is obtained at input diameter of $15 \mathrm{~mm}$. This can be attributed to the variation of the electric field inside the accelerating tube.

Figure $5 \mathrm{a}$ and $\mathrm{b}$ show the influence of the voltage ratio on both the beam emittance and beam diameter of the argon ion trajectories passed through the accelerator system. It was seen that from this figure, minimum beam emittance and beam diameter is obtained, at $4 \mathrm{~cm}$ mrad, $8 \mathrm{~mm}$, respectively. This can be attributed to the variation of the electric field inside the accelerating tube. Figure 6 $a$ and $b$ show the influence of the inner tube diameter on 


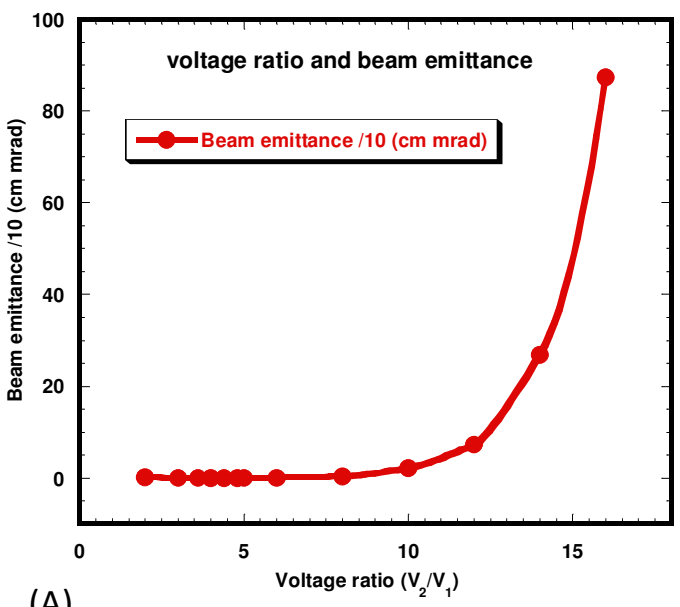

(A)

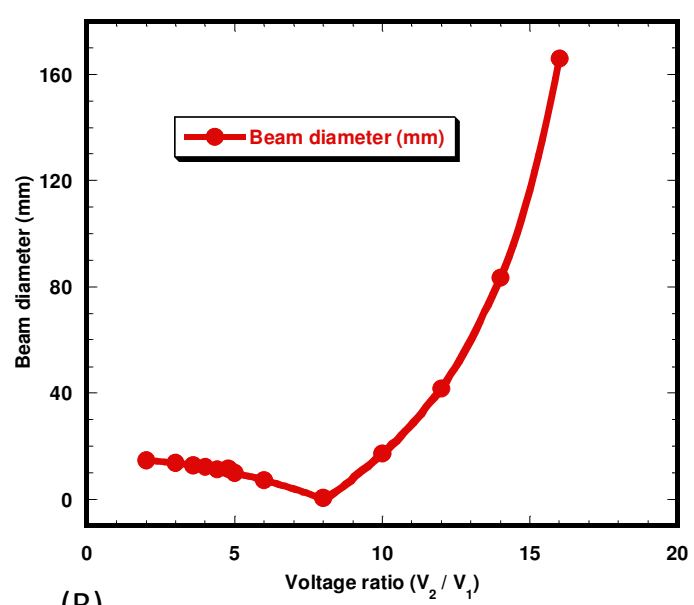

(B)

Figure 5. Influence of the voltage ratio on both beam emittance and beam diameter of the accelerator system for singly charged argon ion trajectories with space charge of $0.1 \mathrm{~mA}$. (A) Beam emittance with voltage ratio. (B) Beam diameter with voltage ratio.

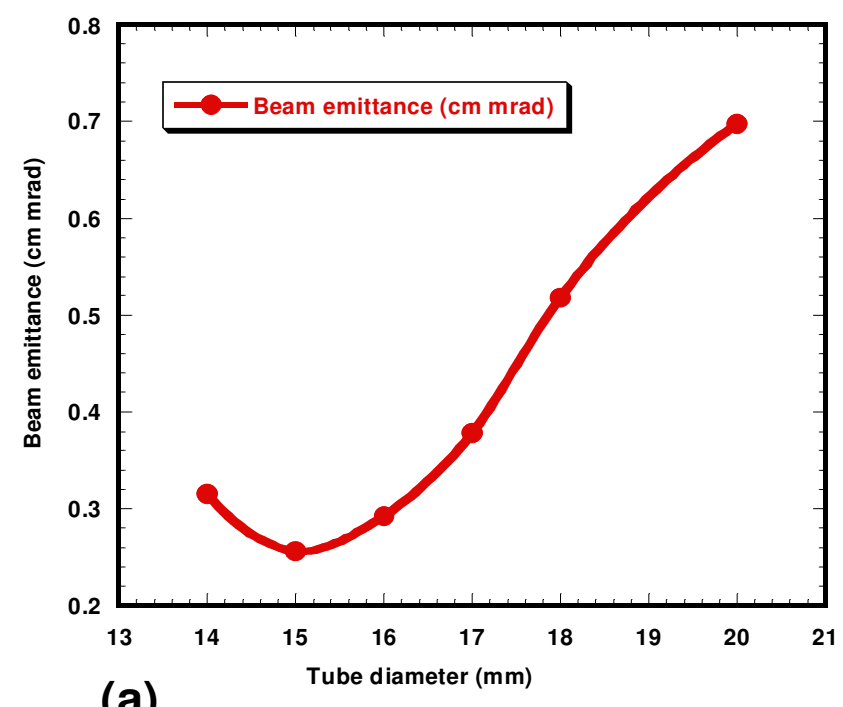

(a)

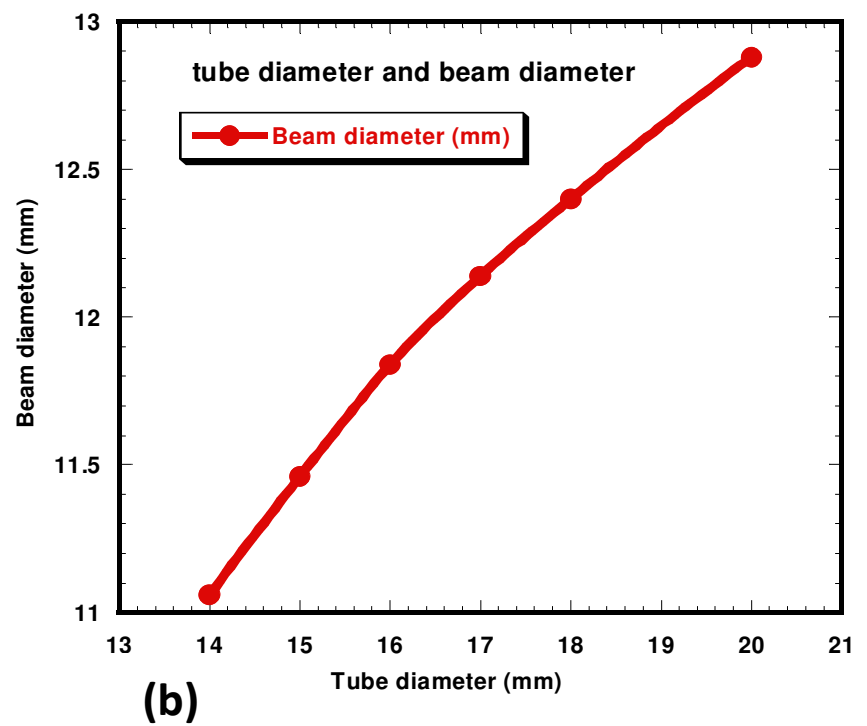

(b)

Figure 6. Beam emittance and beam diameter as a function of the diameter of the accelerator system (A) Beam emittance with tube diameter and (B) Beam diameter with tube diameter.

both the beam emittance and beam diameter of the argon ion trajectories passed through the accelerator system. It was found that, minimum beam emittance at diameter of $15 \mathrm{~mm}$ is gotten. An increase of the inner tube diameter was accompanied by an increase of the beam diameter.

Space charge depends on the geometry of the electrodes, applied potentials and ion current. Therefore, the change of the ion current has a clear influence where other parameters were fixed. The space charge force charge repel each other. It was found that space charge has no influence on the ion beam envelope at currents of micro amperes.

The space charge started to have a clear influence on the ion beam envelope at currents of $1 \times 10^{-4} \mathrm{~A}$ (Figure 7 a, b). Space charge depends on the geometry of the electrodes, applied potentials and ion current. Therefore, the change of the ion current has a clear influence where other parameters were fixed. The space charge force acts as a diverging force because particles of the same charge repel each other. 


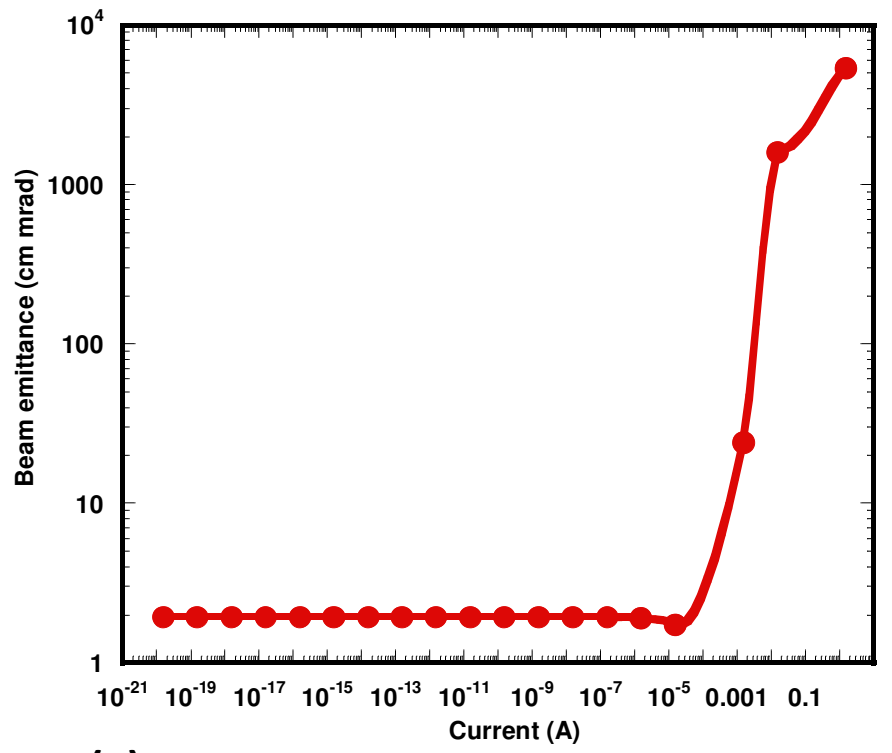

(a)

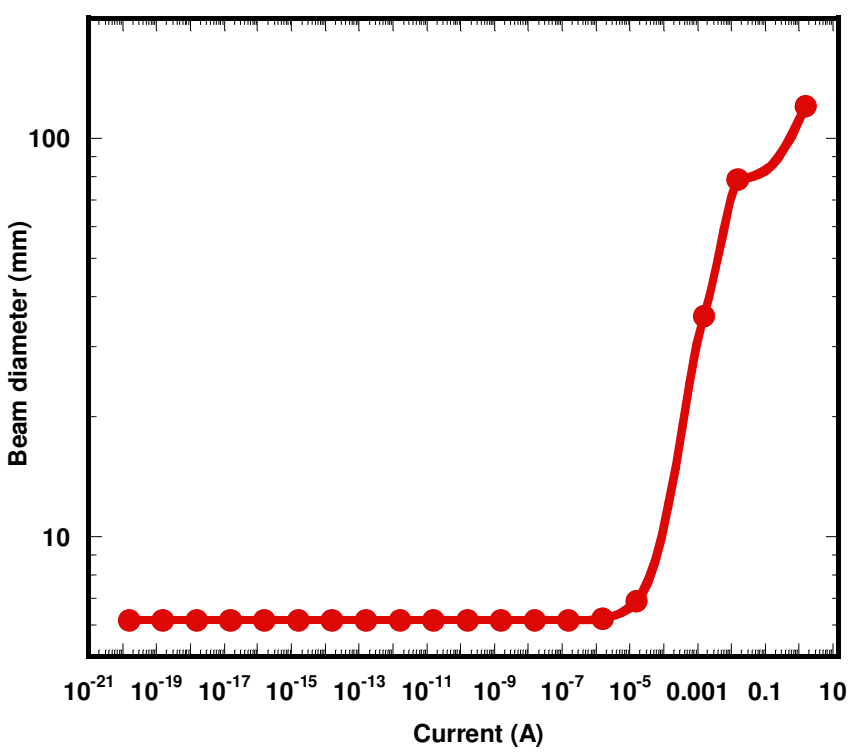

(b)

Figure 7. Influence of space charge on both beam emittance and beam diameter of the accelerator system for singly charged argon ion trajectories (a) Beam emittance with space charge and (b) Beam diameter with space charge.

Influence of the charge states of argon atom on both the beam emittance and beam diameter for the accelerator system has been investigated with current of $0.1 \mathrm{~mA}$ (Figure $8 \mathrm{a}, \mathrm{b}$ ). Minimum beam emittance has been obtained for argon of highly charged ions (charge $=5^{+}$). The same behaviour was found for the beam diameter where, minimum beam diameter was obtained for a highly charged argon ions (charge $=5^{+}$).

Influence of atomic masses of different elements (hydrogen, helium, lithium, carbon, nitrogen, oxygen, neon and finally argon) on both the beam emittance and beam diameter for the single gap tube accelerator system has been investigated (Figure $9 \mathrm{a}, \mathrm{b}$ ). It was found that, an increase of the atomic masses of the chosen elements was accompanied by an increase of the beam emittance and then started to have a minimum for argon.

Whereas for beam diameter, an increase of the chosen elements was accompanied by a decrease of the beam diameter and started to have a minimum for carbon and then increased to the highest beam diameter and finally have a minimum point for argon atom.

\section{Conclusion}

Study of optical ion beam properties for a single gap accelerating column was done by using a SIMION computer program. A series of simulation studies were made in order to determine optimum conditions for acceleration of space-charge-dominated argon ion trajectories with incident energy of $50 \mathrm{keV}$ from a distance $150 \mathrm{~mm}$ of the ion source at constant gradient acceleration system. The new achievement given by both beam simulation and analytical treatment are useful tools for designing of constant gradient acceleration system. The influence of the voltage ratio on both the beam emittance and beam diameter of the argon ion trajectories passed through the accelerator system was done. It was seen that, minimum beam emittance and beam diameter is obtained, at $4 \mathrm{~cm} \mathrm{mrad,} 8 \mathrm{~mm}$, respectively. Also, the influence of the inner tube diameter on both the beam emittance and beam diameter of the argon ion trajectories passed through the accelerator system. It was found that, minimum beam emittance at diameter of $15 \mathrm{~mm}$ is gotten. An increase of the inner tube diameter was accompanied by an increase of the beam diameter. Furthermore, influence of the charge states of argon atom on both the beam emittance and beam diameter for the accelerator system has been investigated with current of $0.1 \mathrm{~mA}$. Minimum beam emittance was obtained for argon of highly charged ions is studied for the accelerator system. Minimum beam emittance is found at a current of $10^{-4} \mathrm{~A}$, and started to have a large effect at current higher than $10^{-4} \mathrm{~A}$. Minimum beam diameter for the system is also found at a current of $10^{-5} \mathrm{~A}$, and is started to have a large effect at current higher than $10^{-5} \mathrm{~A}$. Influence of atomic masses of different elements on both the beam emittance and beam for the accelerator system is investigated. Minimum beam emittance for accelerator system is obtained for argon ( $m$ 


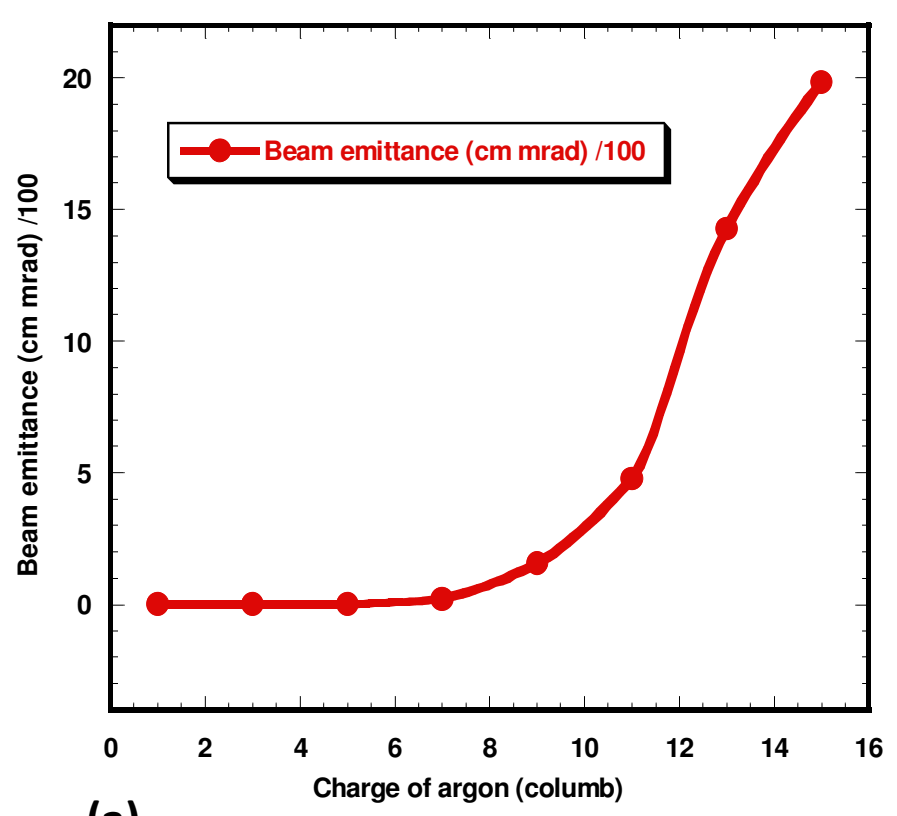

(a)

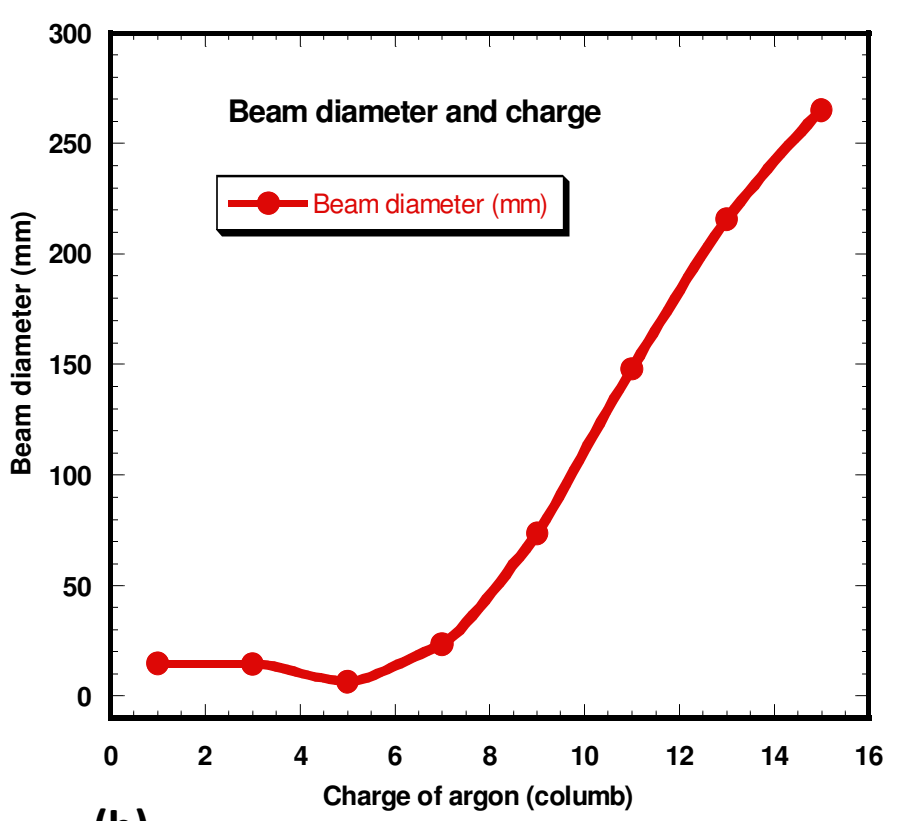

(b)

Figure 8. Beam emittance and beam diameter as a function of the charge states of argon atom (a) Beam emittance with charge and (b) Beam diameter with charge

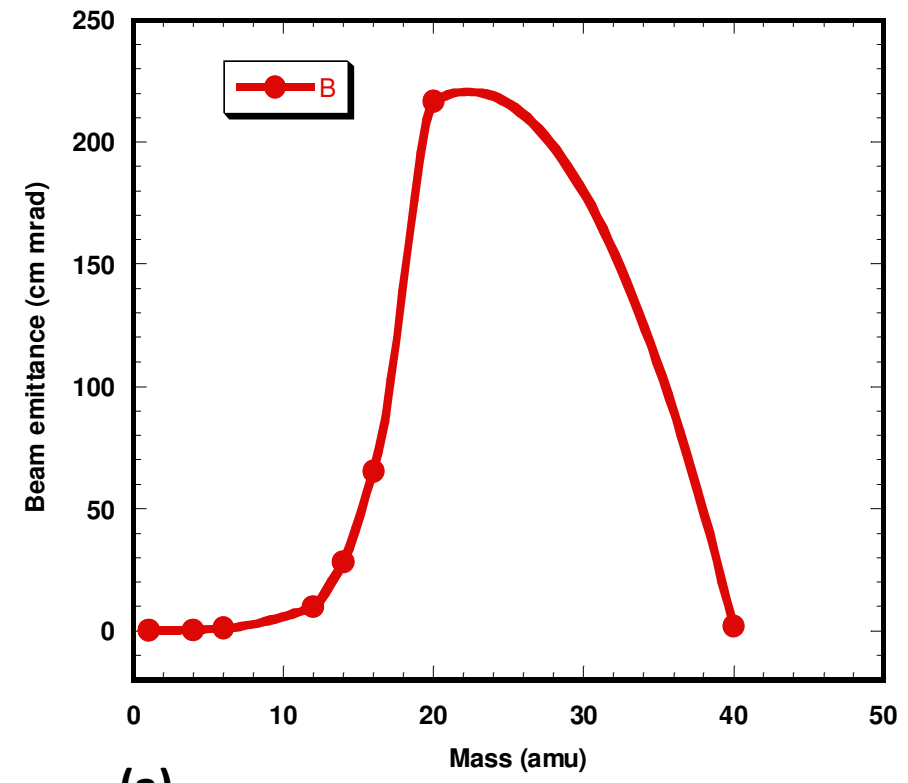

(a)

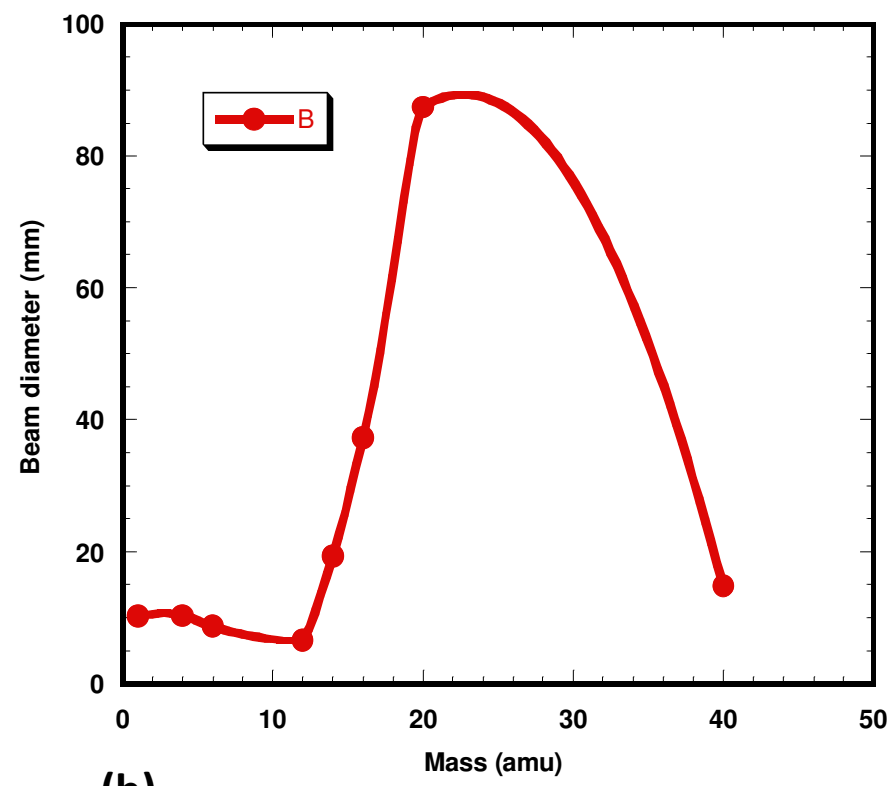

(b)

Figure 9. Beam emittance and beam diameter as a function of atomic mass for different elements (a) Beam emittance with atomic mass and (b) Beam diameter with atomic mass.

$=40)$. The influences of inner tube diameter and accelerating voltage ratio slightly have the same effect on output beam emittance.

\section{ACKNOWLEDGMENTS}

Authors are grateful to both reviewers for their valuable 
comments towards improving the quality of the work. The authors gratefully acknowledge Prof. M. E. Abdelaziz, ExPresident of Egypitation Atomic Energy Authority, Egypt for his valuable discussions and scientific help.

\section{REFERENCES}

Abdelaziz ME, Zakhary SG, Ghanem AA (1996). Factors affecting the design of a single gap accelerating column, "Rev. Sci. Instrum." 67:3.

Abdelrahman MM, Zakhary SG (2011). Proposed design of DC low voltage acceleration system. Int. J. Phys. Sci. 6:5492-5497.

Dahl DA (2000). SIMION 3 D Version 7.0 User's Manual INEEL-95 / 0403, Idaho National Engineering and Environmental Laboratory; I D 83415.

Dahl DA, Delmoreand JE, Appelhans AD (1990). SIMION PC/PS2 electrostatic lens design program. Rev. Sci. Instrum. 61:607.

Humphries S (2002), Charged Particle Beams, John Wiley and Sons, USA.

Kirstein PT, Kino CS, Waters WE (1967). Space Charge Flow, McGrawHill, New York.
Kunze HJ, El-Khalafawy T, Hegazy H (2003).First Cairo conf. on plasma physics and applications. p. 34.

Lee SY (2000). Accelerator Physics, World Scientific Publishing Co. Pte. Ltd, 5 Toh Tuck Link, Singapore 596224.

Litovko I, Oks E (2006). 33rd EPS Conference on Plasma Phys. Rome, 19 - 23 June ECA .30I(2):104.

Prakash NP, Amit RP (2012). Design of a superconducting low beta niobium resonator J. Phys. 78:4.

Reiser M (1985). Particle Accelerator Conf., Accelerator Engineering and Technology, Vancouver, Canada. p. 3.

Spadtke P (1992), Numerical simulation of ion beam related problems, Rev. Sci. Instrum. 63:(4)2647-2651.

Spakudo P, Hashi K (1996). Exact energy values of "low-energy ion beams' Rev. Sci. Instrum. 67:3.

Wilson RG, Brewer GR (1973). "Ion Beams with Applications to Ion Implantation", John Wiley and Sons, New York. 